\title{
CANONICAL STRETCHED RINGS
}

\author{
NGUYEN THI ANH HANG, DO VAN KIEN, AND HOANG LE TRUONG \\ Dedicated to Professor Nguyen Tu Cuong on the Occasion of His 70th Birthday
}

\begin{abstract}
In this paper, we introduce the concept of canonical stretched rings, sparse stretched rings and maximum sparse ideals. Then we give characterizations of canonical stretched rings and sparse stretched rings; and a characterization of Gorenstein rings in terms of their maximum sparse ideals. Several explicit examples are provided along the paper to illustrate such rings.
\end{abstract}

\section{INTRODUCTION}

This paper studies a special class of Cohen-Macaulay local rings, which we call canonical stretched rings. Originally, the definition of stretched Cohen-Macaulay rings was introduced by Sally in 1979 (cf. [Sal79]). She developed in [Sal79] a very nice theory of stretched CohenMacaulay rings and gave many interesting results such as a structure theorem for stretched Artinian local rings ([Sal79, Theorem 1.1] and cf. [EV08, Theorem 3.1]). Recall that an Artinian local ring $(R, \mathfrak{m})$ is called a stretched ring, if $\mathfrak{m}^{2}$ is principal. After that, Sally formulated the notion of "stretched" for Cohen-Macaulay local rings of higher dimension in terms of a minimal reduction of $\mathfrak{m}$. In particular, a Cohen-Macaulay local ring $(S, \mathfrak{n})$ is said to be stretched, if there is a minimal reduction $\mathfrak{q}$ of $\mathfrak{n}$ such that $\mathfrak{q}$ is a parameter ideal of $S$ and $S / \mathfrak{q}$ is a stretched Artinian local ring. In this paper, we give the new notion of "stretched" for Cohen-Macaulay local rings in arbitrary dimension in terms of their irreducible ideals. Notice that an ideal $I$ of $S$ is said to be irreducible, if $I$ is not written as the intersection of two larger ideals of $S$. We call a Cohen-Macaulay local ring $(S, \mathfrak{n})$ to be a canonical stretched ring, if there is an irreducible ideal $I \subseteq \mathfrak{n}^{2}$ of $S$ such that $S / I$ is a stretched Artinian local ring.

Now let us explain our notation, terminology and motivation about canonical stretched rings. Assume that $(S, \mathfrak{n})$ is a one-dimensional Cohen-Macaulay local ring possessing a canonical module $\omega_{S}$. We say that an ideal $I$ of $S$ is canonical, if $I \neq S$ and $I \cong \omega_{S}$ as $S$-modules. It is well-known that because canonical ideals are faithful $S$-module [HK71, Bemerkung 2.5], they are n-primary. Conversely, also by [HK71, Satz 3.3], an n-primary ideal $I$ is a canonical ideal of $S$ if and only if $I$ is an irreducible ideal of $S$. Then our definition of canonical stretched rings is now stated as follows.

Key words and phrases. Cohen-Macaulay local ring, Canonical ideals, Stretched Cohen-Macaulay rings. 2020 Mathematics Subject Classification: 13H10, 13 H15. 
Definition 1.1. We say that $(S, \mathfrak{n})$ is a canonical stretched ring, if $S$ is a one-dimensional Cohen-Macaulay local ring possessing a canonical ideal $I \subseteq \mathfrak{n}^{2}$ such that $S / I$ is a stretched ring.

Let us explain how this paper is organized. In Section 2, we revisit some well-known results about stretched rings and canonical ideals. In Section 3, we shall give characterizations of canonical stretched rings. In Section 4, we shall introduce the notion of maximum sparse ideals in one-dimensional Noetherian local domains and give a characterization of Gorenstein rings in terms of their maximum sparse ideals. Moreover we will study a special class of canonical stretched rings, which we call sparse stretched rings. In the final section, we will explore 3-generated numerical semigroup rings over a field and their canonical stretched property.

\section{Notation, CONVEntions, AND PRELIMINARY RESUlts}

In this section, we shall summarize preliminary results, which we need throughout this paper. Some of them are known but we will provide short proofs for the sake of completeness.

2.1. "Let $(R, \mathfrak{m}, k)$ be a local ring" identifies $\mathfrak{m}$ as the unique maximal ideal of the commutative Noetherian local ring $R$ and $k$ as the residue field $k=R / \mathfrak{m}$.

(1) The embedding dimension of $R$ is defined to be $\operatorname{edim}(R)=\operatorname{dim}_{k}\left(\mathfrak{m} / \mathfrak{m}^{2}\right)$.

(2) The Hilbert function $h_{R}$ of $R$ is defined by

$$
h_{R}(i)=\ell_{R}\left(\mathfrak{m}^{i} / \mathfrak{m}^{i+1}\right) \quad \text { for all } i \geq 0 .
$$

(3) The function $h_{R}$ is called non-decreasing if $h_{R}(n-1) \leq h_{R}(n)$ for each $n \in \mathbb{N}$ and decreasing if there exists $\ell \in \mathbb{N}$ such that $h_{R}(\ell-1)>h_{R}(\ell)$, in this case we say $h_{R}$ decreases at level $\ell$.

(4) If $M$ is an $R$-module, then the socle of $M$ is the vector space $\operatorname{Soc}(M)=0:_{M} \mathfrak{m}$, $\mu_{R}(M)$ denotes the minimal number of generators of $M$ and we denote by $\ell_{R}(M)$ its length.

(5) Let $I$ be an $\mathfrak{m}$-primary ideal of $R$. Then the top socle degree of $I$ is defined to be the maximum integer $s$ such that $(I: \mathfrak{m}) \cap\left(\mathfrak{m}^{s}+I\right) \neq(I: \mathfrak{m}) \cap\left(\mathfrak{m}^{s+1}+I\right)$ and denoted by $s(I)$. In particular, if $(R, \mathfrak{m}, k)$ is an Artinian local ring, then the top socle degree of $R$ is defined to be the maximum integer $s$ with $\mathfrak{m}^{s} \neq 0$, and denoted by $s(R)$.

(6) The Hilbert-Samuel function $H_{R}$ of $R$ is defined by $H_{R}(n)=\ell_{R}\left(R / \mathfrak{m}^{n+1}\right)$ for each $n \in \mathbb{N}$.

(7) If $I$ is an ideal of $R$, then $v_{R}(R / I)=v_{R}(I)=\max \left\{n \mid I \subseteq \mathfrak{m}^{n}\right\}$.

For each non-zero element $a \in R$, we define the degree of $a$ with respect to $\mathfrak{m}$ by $\operatorname{deg} a=$ $\max \left\{n \geq 0 \mid a \in \mathfrak{m}^{n}\right\}$. Then the top socle degree of $I$ can be computed through the degree of an element in the socle of $R / I$, as follows.

Proposition 2.1. Let $(R, \mathfrak{m})$ be a local ring and $I$ an $\mathfrak{m}$-primary ideal. Then we have

1) $s(I)=\max \{\operatorname{deg} a \mid a \in(I: \mathfrak{m}) \backslash I\}$,

2) $(I: \mathfrak{m}) \cap \mathfrak{m}^{s(R)} \backslash I=\{a \in(I: \mathfrak{m}) \backslash I \mid \operatorname{deg} a=s(R)\}$. 
Proof. 1) Suppose that there exists $a \in(I: \mathfrak{m}) \backslash I$ such that $\operatorname{deg} a>s(I)$. Then

$$
(I: \mathfrak{m}) \cap \mathfrak{m}^{\operatorname{deg}(a)}+I=(I: \mathfrak{m}) \cap \mathfrak{m}^{\operatorname{deg}(a)+i}+I \text { for all } i \geq 1 .
$$

Hence $(I: \mathfrak{m}) \cap \mathfrak{m}^{\operatorname{deg}(a)}+I=I$ which implies that $(I: \mathfrak{m}) \cap \mathfrak{m}^{\operatorname{deg}(a)} \subseteq I$. Hence, since $a \in(I: \mathfrak{m}) \backslash I$, we get $a \notin \mathfrak{m}^{\operatorname{deg}(a)}$ which is a contradiction. Therefore, $\operatorname{deg}(a) \leq s(I)$ for all $a \in(I: \mathfrak{m}) \backslash I$. To show that there is an element $a \in(I: \mathfrak{m}) \backslash I \operatorname{such}$ that $\operatorname{deg}(a)=s(I)$, it is sufficient to prove 2).

2) It is clear that the left hand side contains the right hand side. For reverse inclusion, take any $a \in(I: \mathfrak{m}) \cap \mathfrak{m}^{s(I)} \backslash I$. We then have $a \in(I: \mathfrak{m}) \backslash I$. Moreover, by the proof of 1$)$ we get $\operatorname{deg}(a) \leq s(I)$. On the other hand, since $a \in \mathfrak{m}^{s(I)}$, we have $\operatorname{deg}(a) \geq s(I)$. Therefore, $\operatorname{deg}(a)=s(I)$, which implies that the left hand side is contained in the right hand side, as required.

2.2. The definition of stretched local rings was first introduced by Sally in 1979 (cf. [Sal79]).

Definition 2.2. (cf. [Sal79]) We say that a $d$-dimensional local ring $(S, \mathfrak{n})$ is stretched, if there is a minimal reduction $J$ of $\mathfrak{n}$ such that $J$ is a parameter ideal of $S$ and $\ell_{S}\left(\frac{\mathfrak{n}^{2}+J}{\mathfrak{n}^{3}+J}\right)=1$.

If $R$ is an Artinian local ring, by the classical theorem of Macaulay on the shape of the Hilbert function of a standard graded algebra, the Hilbert function of a stretched Artinian local ring $R$ is given by:

\begin{tabular}{|c|c|c|l|c|c|}
\hline 0 & 1 & 2 & $\cdots$ & $s(R)$ & $s(R)+1$ \\
\hline 1 & $\operatorname{edim}(R)$ & 1 & $\cdots$ & 1 & 0 \\
\hline
\end{tabular}

2.3. Suppose that $(S, \mathfrak{n})$ is a Cohen-Macaulay local $\operatorname{ring}$ with $\operatorname{dim} S=1$. Let $\omega_{S}$ denote the canonical module of $S$. Recall that for the $\mathfrak{n}$-adic completion $\hat{S}$ of $S$, the canonical module $\omega_{\hat{S}}$ of $\hat{S}$ is defined by

$$
\omega_{\hat{S}}:=\operatorname{Hom}_{\hat{S}}\left(H_{\mathfrak{n}}^{1}(\hat{S}), \hat{E}\right),
$$

where $H_{\mathfrak{n}}^{1}(\hat{S})$ denotes the first local cohomology module of $\hat{S}$ with respect to $\hat{\mathfrak{n}}$ and $\hat{E}=$ $E_{\hat{S}}(\hat{S} / \hat{\mathfrak{n}})$ the injective envelope of the $\hat{S}$-module $\hat{S} / \hat{\mathfrak{n}}$. When $S$ is not necessarily $\mathfrak{n}$-adically complete, the canonical module $\omega_{S}$ of $S$ is defined to be an $S$-module such that

$$
\hat{S} \otimes_{S} \omega_{S} \cong \omega_{\hat{S}}
$$

as $S$-modules.

The fundamental theory of canonical modules was developed in [HK71] by J. Herzog and E. Kunz. It is well-known that $S$ possesses the canonical module $\omega_{S}$ if and only if $S$ is a homomorphic image of a Gorenstein ring. In the present research, we are interested in rings which contain canonical ideals. Let us begin with the following.

Definition 2.3. An ideal $I$ of $S$ is said to be a canonical ideal of $S$, if $I \neq S$ and $I \cong \omega_{S}$ as $S$-modules.

Here we remark that this definition implicitly assumes the existence of the canonical module $\omega_{S}$. Namely, the condition in Definition 2.3 that $I \cong \omega_{S}$ as $S$-modules should be read to mean that $S$ possesses the canonical module $\omega_{S}$ and the ideal $I$ of $S$ is isomorphic to $\omega_{S}$ as $S$-modules. We then have the following result. 
Lemma 2.4. Let $(S, \mathfrak{n})$ be a local ring of dimension one, $Q$ its total ring of fractions and $\bar{S} \subseteq Q$ its normalization. Assume that $S$ possesses a canonical module. Then we have

1) $S$ has a canonical ideal if and only if the total ring of fractions of $\hat{S}$ is Gorenstein. ([GMP13, HK71]).

2) An $\mathfrak{n}$-primary ideal $I$ is a canonical ideal if and only if $I$ is an irreducible ideal. ([HK71, Satz 3.3]).

3) There is a canonical module $\omega_{S}$ of $S$ such that $\omega_{S}: Q \bar{S}=S: Q \bar{S}$ and $S \subseteq \omega_{S} \subseteq$ $\bar{S} \cdot([\mathrm{BH} 92$, Lemma 3$])$.

\section{CAnonical Stretched Rings}

3.1. Now we begin by setting the notation of this section.

Setting 3.1. Let $(S, \mathfrak{n}, k)$ be a commutative Noetherian local ring with the maximal ideal $\mathfrak{n}$ and $\operatorname{dim} S=d$. Let $I$ be an $\mathfrak{n}$-primary ideal of $S$ such that $(R:=S / I, \mathfrak{m}:=\mathfrak{n} / I, k)$ is an Artinian local ring with the top socle degree $s$.

The following lemma give the basic properties of the top socle degree and the invariant $v_{S}(R)$ (see 2.1).

Lemma 3.2. Let $S$ and $R$ be as in Setting 3.1. Then we have

1) $s(R)=\max \left\{i \in \mathbb{N} \mid \mathfrak{n}^{i}+I \neq \mathfrak{n}^{i+1}+I\right\}$.

2) $v_{S}(R)=\inf \left\{i \mid h_{R}(i)<h_{S}(i)\right\}$.

Proof. 1) Let $t=\max \left\{i \in \mathbb{N} \mid \mathfrak{n}^{i}+I \neq \mathfrak{n}^{i+1}+I\right\}$. It is clear that $s(R) \leq t$. Assume that $s(R)<t$. Then $(I: \mathfrak{n}) \cap \mathfrak{n}^{t}+I=(I: \mathfrak{n}) \cap \mathfrak{n}^{t+1}+I$. By Nakayama's lemma, we get $\mathfrak{n}^{t}+I=\mathfrak{n}^{t+1}+I$ which is impossible. We therefore have $t=s(R)$.

2 ) It is now immediate from the definition of $v_{S}(R)$.

Recall that in the case of Cohen-Macaulay algebras, Ooishi gave the definition of stretched Cohen-Macaulay algebras in terms of their Castelnuovo-Mumford regularity ([Ooi82, Definition 14]). In particular, if $A$ is a homogeneous Cohen-Macaulay algebra over a field $k$ then

$$
\operatorname{reg}(A) \leq e(A)+\operatorname{dim}(A)-\operatorname{edim}(A),
$$

where $\operatorname{reg}(A)$ is the Castelnuovo-Mumford regularity of $A, e(A)$ is the multiplicity of $A$, and $\operatorname{edim}(A)$ is the embedding dimension of $A$. Then $A$ is called a stretched Cohen-Macaulay algebra, if equality holds in 3.2.1. Therefore, if $(S, \mathfrak{n})$ is a local ring such that its associated graded $\operatorname{ring} G(S):=\bigoplus_{n \geq 0} \mathfrak{n}^{n} / \mathfrak{n}^{n+1}$ is Cohen-Macaulay, then $G(S)$ is a stretched Cohen-Macaulay algebra exactly when $S$ is a stretched local ring in the sense of Sally. Notice that if $\operatorname{dim} A=0$ then the Castelnuovo-Mumford regularity of $A$ is the top socle degree of $A$. In the present paper, the following theorem give a bound for the top socle degree and we use such bound to define canonical stretched rings (Definition 3.6).

Theorem 3.3. We have

$$
s(R) \leq \ell_{S}(R)-\sum_{i=0}^{v_{S}(R)-1} h_{S}(i)+v_{S}(R)-1,
$$


and if $I$ is not a power of $\mathfrak{n}$ then the following conditions are equivalent.

1) $s(R)=\ell_{S}(R)-\sum_{i=0}^{v_{S}(R)-1} h_{S}(i)+v_{S}(R)-1$.

2) $\mu\left(\mathfrak{m}^{v_{S}(R)}\right)=1$.

3) The Hilbert function of $R$ is given by

\begin{tabular}{|c|c|l|c|c|l|c|c|}
\hline 0 & 1 & $\cdots$ & $v_{S}(R)-1$ & $v_{S}(R)$ & $\cdots$ & $s(R)$ & $s(R)+1$ \\
\hline 1 & $h_{R}(1)$ & $\cdots$ & $h_{R}\left(v_{S}(R)-1\right)$ & 1 & $\cdots$ & 1 & 0 \\
\hline
\end{tabular}

Proof. To prove the inequality, we put $t=\ell_{S}(R)-\sum_{i=0}^{v_{S}(R)-1} h_{S}(i)$. Then

$$
\begin{aligned}
t=\ell_{S}(S / I)-\sum_{i=0}^{v_{S}(R)-1} h_{S}(i) & =\ell_{S}(S / I)-H_{S}\left(v_{S}(R)-1\right) \\
& =\ell_{S}(S / I)-\ell_{S}\left(S / \mathfrak{n}^{v_{S}(R)}\right)=\ell_{S}\left(\mathfrak{n}^{v_{S}(R)} / I\right) .
\end{aligned}
$$

Moreover, by the definition of $v_{S}(R), I \subseteq \mathfrak{n}^{v_{S}(R)}$. By taking a composition series

$$
I=I_{t} \subsetneq I_{t-1} \subsetneq \ldots \subsetneq I_{0}=\mathfrak{n}^{v_{S}(R)},
$$

we get $I_{i} / I_{i+1} \cong S / \mathfrak{n}$ for all $0 \leq i \leq t-1$. Therefore, $\mathfrak{n}^{t+v_{S}(R)} \subseteq I$. Hence, we obtain $s(R) \leq t+v_{S}(R)-1$ as required.

We now assume that $I$ is not a power of $\mathfrak{n}$. The implication (3) to (2) is obvious. In the following, we give a proof of 1) to 3), 2) to 3), and 3) to 1).

$1) \Rightarrow 3)$ Suppose the first condition holds, that is, $t=s(R)-v_{s}(R)+1$. Then, since $t=\ell_{S}\left(\mathfrak{n}^{v_{S}(R)} / I\right)=\ell_{R}\left(\mathfrak{m}^{v_{S}(R)}\right)$ and $v_{S}(R) \leq s(R)$ (by $I$ is not a power of $\mathfrak{n}$ ), we have

$$
\sum_{i=v_{S}(R)}^{s(R)} \ell_{R}\left(\mathfrak{m}^{i} / \mathfrak{m}^{i+1}\right)=s(R)-v_{s}(R)+1 .
$$

Hence, since $\ell_{R}\left(\mathfrak{m}^{i} / \mathfrak{m}^{i+1}\right) \geq 1$ for all $i \leq s(R)$, we get

$$
\ell_{R}\left(\mathfrak{m}^{i} / \mathfrak{m}^{i+1}\right)=1
$$

for all $v_{S}(R) \leq i \leq s(R)$. Therefore, by definition of $v_{S}(R)$, the Hilbert function of $R$ is given by (3.3.2).

$2) \Rightarrow 3$ ) Assume that $\mu\left(\mathfrak{m}^{v_{S}(R)}\right)=1$. Then, by the classical theorem of Macaulay on the shape of the Hilbert function of a standard graded algebra, we also have $\ell_{R}\left(\mathfrak{m}^{i} / \mathfrak{m}^{i+1}\right)=1$ for all $v_{S}(R) \leq i \leq s(R)$. We conclude that the Hilbert function of $R$ is given by (3.3.2).

$3) \Rightarrow 1$ ) Suppose the Hilbert function of $R$ is given by (3.3.2). Then we have

$$
\ell_{S}(R)=\sum_{i=0}^{v_{S}(R)-1} h_{S}(i)+s(R)-v_{S}(R)+1,
$$

which implies that the equality holds in (3.3.1). 
Corollary 3.4. Assume that $v_{S}(R) \geq 2$. Then we have

$$
s(R) \leq \ell_{S}(R)-\operatorname{edim}(R) .
$$

If $I$ is not a power of $\mathfrak{n}$ then the equality holds in (3.4.1) if and only if $R$ is a stretched Artinian local ring. In this case, we have

$$
v_{S}(R)=2 \text { and } \mu\left(\mathfrak{m}^{2}\right)=1 .
$$

Proof. Since $v_{S}(R) \geq 2, I \subseteq \mathfrak{n}^{2}$. We have $\operatorname{edim}(R)=\operatorname{dim}_{k} \mathfrak{n} / \mathfrak{n}^{2}=h_{S}(1)$. Assume that there exists $0 \leqslant i \leqslant v_{S}(R)-1$ such that $h_{S}(i)=0$. Then we have $\mathfrak{n}^{i}=(0)$ which implies $\mathfrak{n}^{v_{S}(R)}=(0)$. Hence, by $I \subseteq \mathfrak{n}^{v_{S}(R)}, I=(0)$ which is impossible. Therefore, $h_{S}(i) \geqslant 1$ for all $0 \leqslant i \leqslant v_{S}(R)-1$. It follows that

$$
v_{S}(R)-1+\operatorname{edim}(R) \leq \sum_{i=0}^{v_{S}(R)-1} h_{S}(i) .
$$

Hence, by Theorem 3.3, we get

$$
s(R) \leq \ell_{S}(R)-\sum_{i=0}^{v_{S}(R)-1} h_{S}(i)+v_{S}(R)-1 \leq \ell_{S}(R)-\operatorname{edim}(R)
$$

which proves the inequality in (3.4.1).

Now we assume that $R$ is a stretched Artinian local ring in the sense of Definition 2.2, then the Hilbert function of $R$ is given by (2.2.1). Therefore, we have

$$
s(R)=\sum_{i=0}^{s(R)} h_{R}(i)-\operatorname{edim}(R)=\ell_{S}(R)-\operatorname{edim}(R),
$$

as required.

Conversely, we assume that the equality holds in (3.4.1). Then, by (3.4.3), we have

$$
-\sum_{i=0}^{v_{S}(R)-1} h_{S}(i)+v_{S}(R)-1=-\operatorname{edim}(R) .
$$

Since $\operatorname{edim}(R)=h_{S}(1)$, we have $h_{S}(0)+\sum_{i=2}^{v_{S}(R)-1} h_{S}(i)+1=v_{S}(R)$. Since $h_{S}(i) \geq 1$ for all $0 \leq i \leq v_{S}(R)$, we must have $v_{S}(R)=2$. By Theorem 3.3, we have $\mu\left(\mathfrak{m}^{2}\right)=1$. Hence, $R$ is a stretched Artinian local ring, as required.

Example 3.5. Let $k[[t]]$ be the formal power series ring over a field $k$.

1) Let $S=k\left[\left[t^{3}, t^{7}, t^{8}\right]\right], I=\left(t^{6}, t^{7}\right)$, and $\mathfrak{n}=\left(t^{3}, t^{7}, t^{8}\right)$. Then $I$ is an $\mathfrak{n}$-primary ideal of $S$. Put $R=S / I$. Observe that $S / I$ has the length 4 and embedding dimension $\operatorname{edim}(S / I)=2$. We have $\mathfrak{n}^{3} \subseteq I$. It implies that $0<s(R) \leq 2$. Moreover, because the complement $\left((I: \mathfrak{n}) \cap \mathfrak{n}^{2}+I\right) \backslash\left((I: \mathfrak{n}) \cap \mathfrak{n}^{3}+I\right)$ is not empty (it contains $\left.t^{11}\right)$, we have $s(R) \geq 2$. Hence $s(R)=2=\ell_{S}(S / I)-\operatorname{edim}(S / I)$. Therefore, $S$ is a stretched ring. 
2) Let $e \geq 3$ be an arbitrary integer. We consider the local ring

$$
S=k\left[\left[t^{e}, t^{e+1}, \ldots, t^{2 e-1}\right]\right]
$$

in the formal power series ring $k[[t]]$. Let $I=\left(t^{e}, t^{e+1}, \ldots, t^{2 e-2}\right)$ is an $\mathfrak{n}$-primary ideal of $S$. Then $S / I$ has the length 2 and embedding $\operatorname{dimension} \operatorname{edim}(S / I)=1$. We have $\mathfrak{n}^{2} \subseteq I \subsetneq \mathfrak{n}$, whence $0<s(S / I) \leq 1$. We get $s(S / I)=1=\ell_{S}(S / I)-\operatorname{edim}(S / I)$. Hence, $S$ is also a stretched ring.

Now we define canonical stretched rings and give their characterizations.

Definition 3.6. We say that $(S, \mathfrak{n})$ is a canonical stretched local ring, if there is an irreducible ideal $I \subseteq \mathfrak{n}^{2}$ of $S$ such that $R=S / I$ is a stretched Artinian local ring.

We now develop the theory of canonical stretched rings. Let us begin with the following.

Lemma 3.7. Assume that $S$ is a canonical stretched ring. Then, there is an irreducible ideal $I \subseteq \mathfrak{n}^{2}$ of $S$ and a basis $z_{1}, \ldots, z_{\operatorname{edim}(S)}$ for $\mathfrak{n}$ such that

i) $\mathfrak{n}^{n}+I=\left(z_{1}^{n}\right)+I$ for all $n \geq 2$ and $z_{1} z_{i} \in I$ for all $2 \leq i \leq \operatorname{edim}(S)$.

ii) For all $2 \leq i, j \leq \operatorname{edim}(S)$, either $z_{i} z_{j} \in I$ or $z_{i} z_{j}-u_{i j} z_{1}^{s(S / I)} \in I$ for some unit $u_{i j}$ of $S$.

iii) For each $2 \leq i \leq \operatorname{edim}(S)$ there exists $j \geq 2$ such that $z_{i} z_{j}-u_{i j} z_{1}^{s(S / I)} \in I$ for some unit $u_{i j}$ of $S$.

Proof. Since $S$ is a canonical stretched ring, there exists an irreducible ideal $I \subseteq \mathfrak{n}^{2}$ of $S$ such that $S / I$ is a stretched ring. Let $R=S / I, \mathfrak{m}=\mathfrak{n} / I$, and $s=s(R)$. Since $I$ is an irreducible ideal, $R=S / I$ is a Gorenstein ring. In other words, $R=S / I$ is a zero dimensional stretched local Gorenstein ring. Then, by [Sal79, Theorem 1.1], there are elements $z_{1}, z_{2}, \ldots, z_{\text {edim } S}$ in $\mathfrak{n}$ satisfying the following conditions.

a) $\bar{z}_{1}, \ldots, \bar{z}_{\operatorname{edim}(S)}$ is a basis for $\mathfrak{m}$, where $\bar{z}_{i}$ denote the image of $z_{i}$ in $R$ for all $i$.

b) $\left(\bar{z}_{2}, \bar{z}_{3}, \ldots, \bar{z}_{\text {edim } S}\right) \subseteq 0: R \mathfrak{m}^{2}$.

c) $\mathfrak{m}^{n}=\left(\bar{z}_{1}^{n}\right)$ for all $n=2,3, \ldots, \ell_{S}(R)-\operatorname{edim} S$.

d) $\bar{z}_{1} \in\left(0:_{R}\left(\bar{z}_{2}, \ldots, \bar{z}_{\text {edim } S}\right)\right)$.

e) For each $2 \leq i \leq \operatorname{edim} S$ there is $2 \leq j \leq \operatorname{edim} S$ such that $\bar{z}_{i} \bar{z}_{j}=u_{i j} \bar{z}_{1}^{s(R)}$ for some units $u_{i j}$ in $R$.

It follows from $R$ is a stretched ring and Corollary 3.4 that $s=\ell_{S}(R)$ - edim $S$. We therefore get the assertions i) and iii).

Now we show the assertion ii). Indeed, assume that there exists $2 \leq i, j \leq \operatorname{edim}(S) \operatorname{such}$ that $\bar{z}_{i} \bar{z}_{j} \neq 0$. Then by the assertion c) we have $\bar{z}_{i} \bar{z}_{j}=\bar{u}_{i j} \bar{z}_{1}^{n}$, for some $n \geq 2$ and some unit $\bar{u}_{i j} \in R$. On the other hand, by the assertion $\mathrm{d}$ ), we have $\bar{u}_{i j} \bar{z}_{1}^{n+1}=\bar{z}_{1} \bar{z}_{i} \bar{z}_{j}=0$. By the definition of $s$, we have $n=s$. Hence $\bar{z}_{i} \bar{z}_{j}=0$ or $\bar{z}_{i} \bar{z}_{j}=\bar{u}_{i j} \bar{z}_{1}^{s}$ for some unit $u_{i j}$ of $S$. Therefore we get the assertions ii).

Theorem 3.8. Assume that the Hilbert function of $S$ is non-decreasing. Then the following statements are equivalent.

1) $S$ is a canonical stretched ring. 
2) There exists an $\mathfrak{n}$-primary ideal $I \subseteq \mathfrak{n}^{2}$ such that $\mu\left(\mathfrak{n}^{v_{S}(I)} / I\right)=1$ and $I: \mathfrak{n} \subseteq \mathfrak{n}^{v_{S}(I)}$.

When this is the case, $v_{S}(I)=2$.

Proof. 1) $\Rightarrow 2$ ) Since $S$ is a canonical stretched ring, there exists an irreducible ideal $I \subseteq \mathfrak{n}^{2}$ of $S$ such that $S / I$ is a stretched ring. By Corollary 3.4, we have $v_{S}(I)=2, \mu\left(\mathfrak{n}^{v_{S}(I)} / I\right)=1$ and $I: \mathfrak{n} \subseteq \mathfrak{n}^{v_{S}(I)}$.

$2) \Rightarrow 1$ ) Let $R=S / I$ and $\mathfrak{m}=\mathfrak{n} / I$. It follows from $\mu\left(\mathfrak{m}^{v_{S}(I)}\right)=1$ and Corollary 3.4 that the Hilbert function of $R$ is given by 3.3.2. Therefore, by Theorem 3.4 in [Sha14], we have

$$
h_{S}\left(v_{S}(R)-1\right)-h_{S}(1)+1 \leq \operatorname{dim}_{k}\left(0:_{R} \mathfrak{m}\right) .
$$

Moreover, since $0:_{R} \mathfrak{m} \subseteq \mathfrak{m}^{v_{S}(R)}$ and $\mu\left(\mathfrak{m}^{v_{S}(I)}\right)=1$, we have $\operatorname{dim}_{k}\left(0:_{R} \mathfrak{m}\right)=1$. Then $h_{S}\left(v_{S}(R)-1\right)-h_{S}(1) \leq 0$. Since the Hilbert function $h_{S}$ of $S$ is non-decreasing, we have $v_{S}(R)=2$. Then by Corollary $3.4, S / I$ is a stretched ring.

On the other hand, since $\operatorname{dim}_{k}(0: R \mathfrak{m})=1, I$ is an irreducible ideal. Hence, $S$ is a canonical stretched ring.

Example 3.9. Let $k[[X, Y, Z]]$ be the formal power series ring over a field $k$ and

$$
S=k[[X, Y, Z]] /(X, Y) \cap(Y, Z) \cap(Z, X)
$$

the local ring with the maximal ideal $\mathfrak{n}=(x, y, z)$, where $x, y$, and $z$ denote the images of $X, Y$, and $Z$ in $S$, respectively. Then $S$ is a reduced ring but not an integral domain with Cohen-Macaulay type $r(S)=2$ and $\operatorname{dim} S=1$.

Let $s \geq 2, I=\left(x y, y z, z x, x^{s}+y^{2}, x^{s}+z^{2}\right)$ and $R=S / I, \mathfrak{m}=\mathfrak{n} / I$. Then $I \subseteq \mathfrak{n}^{2}$, $I: \mathfrak{n}=\left(x y, y z, z x, x^{s}, y^{2}, z^{2}\right)$ and so $\ell_{S}((I: \mathfrak{n}) / I)=1$. Hence, for all $s \geq 2, I$ is a canonical ideal of $S$. Moreover, the Hilbert functions of $S$ and $R$ are

\begin{tabular}{|c|c|c|c|c|}
\hline 0 & 1 & 2 & 3 & $\ldots$ \\
\hline 1 & 3 & 3 & 3 & $\ldots$ \\
\hline
\end{tabular}$\quad$ and $\quad$\begin{tabular}{|c|c|c|c|c|c|}
\hline 0 & 1 & 2 & $\ldots$ & $s$ & $s+1$ \\
\hline 1 & 3 & 1 & $\ldots$ & 1 & 0 \\
\hline
\end{tabular}

Hence $S$ is a canonical stretched ring.

Example 3.10. Let $e$ be a positive integer, $e \geq 3$. We consider the local ring

$$
S=k\left[\left[t^{e}, t^{e+1}, \ldots, t^{2 e-1}\right]\right]
$$

in the formal power series ring $k[[t]]$ over a field $k$. We put $I=\left(t^{2 e}, t^{2 e+1} \ldots, t^{3 e-2}\right)$. Then $I$ is a canonical ideal of $S$ and Hilbert function of $S / I$ is

$$
h_{S / I}(i)= \begin{cases}1 & \text { if } i=0 \\ e & \text { if } i=1 \\ 1 & \text { if } i=2 \\ 0 & \text { if } i \geq 3\end{cases}
$$

Hence $S$ is a canonical stretched ring. 


\section{MAXIMUm SPARSE IDEALS}

In this section, we study a special class of canonical stretched rings, which we call sparse stretched rings. Let us begin with notations.

A subset $H$ of the set of non-negative integers $\mathbb{N}$ is called a numerical semigroup, if it contains 0 , is closed under addition and has a finite complement in $\mathbb{N}$. The largest integer not belong to $H$ is called the Frobenius number of $H$ and denoted by $g(H)$. We denote $\delta(H):=\sharp(\mathbb{N} \backslash H)$ and call it the genus of the numerical semigroup $H$. Then we obtain an upper bound of the Frobenius number of $H$ by its genus. Indeed, we observe that for all $s \in H$ then $g(H)-s \notin H$. Therefore, $\delta(H) \geq \sharp\{s \in H \mid s<g(H)\}$. But $\sharp\{s \in H \mid s<g(H)\}+\delta(H)=g(H)+1$, we get $g(H) \leq 2 \delta(H)-1$. This result can be extended to the Frobenius number of an ideal in $H$.

We recall that a subset $I$ of a numerical semigroup $H$ is said to be an ideal of $H$, if $I+H \subseteq I$. A relative ideal of $H$ is a subset $F$ of $\mathbb{Z}$ with the property that $F+H \subseteq F$ and $F+h \subseteq H$ for some $h \in H$. An relative ideal $\Omega$ is called a canonical ideal of the semigroup $H$ if $\Omega-(\Omega-F)=F$ for every relative ideal $F$ of $H$. Because the complement of $H$ in $\mathbb{N}$ is finite, it is not difficult to see that the complement of an ideal $I$ of $H$ in $\mathbb{N}$ is finite as well. We also call the largest integer which not belong to the ideal $I$ to be the Frobenius number of $I$ and denote by $g(I)$. We denote the difference of the ideal $I$ with respect to $H$ by $d(I)$, it is exactly the cardinality of $H \backslash I$. In [BA19], M. Bras-Amorós gave an upper bound on the Frobenius number of an ideal which extends the upper bound for the Frobenius number of a numerical semigroup.

Theorem ([BA19, Theorem 1]). Let $I$ be an ideal of a numerical semigroup $H$. Then

$$
g(I) \leq d(I)+2 \delta(H)-1
$$

The ideals for which the Frobenius number attains the bound are called maximum sparse ideals of $H$. In [BA19, Theorem 2] they gave a characterization to a proper ideal that is maximum sparse. It is known that [BALVO14, Theorem 2] the class of maximum sparse ideals of $H$ contains the class of canonical ideals. Moreover, we can use maximum sparse ideals to characterize the symmetry of a numerical semigroup. More precisely, a numerical semigroup $H$ is symmetric if and only if there is a maximum sparse principal ideal [BALVO14, Corollary 1]. In this section, we generalize the notion of maximum sparse ideals to a onedimensional Noetherian local domain.

Setting 4.1. Throughout this section, let $(S, \mathfrak{n})$ be a one-dimensional Noetherian local domain with the infinite residue field $k$ and the quotient field $K$. We assume that $S$ is not regular with normalization $\bar{S} \subseteq K$. We suppose that $\bar{S}$ is a DVR and a finite $S$-module, i.e., $S$ is analytically irreducible. Let $t \in \bar{S}$ be a uniformizing parameter for $\bar{S}$, so that $t \bar{S}$ is the maximal ideal of $\bar{S}$. We also suppose that the field $k$ is isomorphic to the residue field $\bar{S} / t \bar{S}$, i.e., $S$ is residually rational. We denote the usual valuation on $K$ associated to $S$ by Val.

In this setting for a pair of non-zero fractional ideals $J \subseteq I$, it is possible to compute (cf. [Mat71]) the length of the $S$-module $I / J$ by means of valuations, that is,

$$
\ell_{S}(I / J)=|\operatorname{Val}(I) \backslash \operatorname{Val}(J)|
$$


We denote $\operatorname{Val}(S):=\{\operatorname{Val}(a) \mid a \in S, a \neq 0\} \subseteq \mathbb{N}$ to be the value semigroup of $S$. Since the conductor $\mathfrak{C}:=\left(S:_{K} \bar{S}\right)$ is an ideal of both $S$ and $\bar{S}$, there exists a positive integer $c$ so that $\mathfrak{C}=t^{c} \bar{S}, \ell_{S}(\bar{S} / \mathfrak{C})=c$ and $c \in \operatorname{Val}(S)$. Furthermore, we denote $\delta:=\ell_{S}(\bar{S} / S)$ the number of gaps of the semigroup $\operatorname{Val}(S)$. This means that $\delta$ is the genus of $\operatorname{Val}(S)$. Let $r:=\ell_{S}\left((S: \mathfrak{n})_{K} / S\right)$ be the Cohen Macaulay type of $S$. We list the elements of $\operatorname{Val}(S)$ in order of size: $\operatorname{Val}(S):=\left\{s_{i}\right\}_{i \geq 0}$, where $s_{0}=0$ and $s_{i}<s_{i+1}$, for every $i \geq 0$. We put $e:=s_{1}$ the multiplicity of $S$ and $n=c-\delta$ the number such that $s_{n}=c$. For every $i \geq 0$, let $S_{i}$ denote the ideal of elements whose values are bounded by $s_{i}$, that is,

$$
S_{i}:=\left\{a \in S \mid \operatorname{Val}(a) \geq s_{i}\right\} .
$$

Notation 4.2. We assume as in the Setting 4.1. The following is a list of symbols and relations to be used in the sequel. For fractional ideals $I, J$ :

1) $(I: J):=(I: K J)=\{a \in K \mid a J \subseteq I\}$.

2) $\mathfrak{C}_{I}=(I: \bar{S})$, the largest $\bar{S}$-ideal contained in $I$.

Let $I$ be a proper ideal of $S$. We denote

1) $c(I):=\ell_{S}\left(S / \mathfrak{C}_{I}\right)$, so that $t^{c(I)} \bar{S}=\mathfrak{C}_{I} ; c \leq c(I)$ since $\mathfrak{C}_{I} \subseteq \mathfrak{C}$.

2) $n_{I}$ is a number such that $s_{n_{I}}=c(I), \mathfrak{C}_{I}=S_{n_{I}}, n_{I}=\ell_{S}\left(S / \mathfrak{C}_{I}\right)=c(I)-\delta$.

The ideals $S_{i}$ give a strictly decreasing sequence

$$
S=S_{0} \supseteq S_{1}=\mathfrak{n} \nsupseteq S_{2} \supseteq \ldots \nsupseteq S_{n}=\mathfrak{C} \supseteq S_{n+1} \supseteq \ldots,
$$

which induces the chain of duals:

$$
S \varsubsetneqq\left(S: S_{1}\right) \varsubsetneqq \cdots \varsubsetneqq\left(S: S_{n}\right)=\bar{S} \varsubsetneqq\left(S: S_{n+1}\right)=t^{-1} S \varsubsetneqq \cdots
$$

For each non-negative integer $i$, we define $D(i)=\left\{s_{j} \mid s_{j} \leq s_{i}\right.$ and $\left.s_{i}-s_{j} \in \operatorname{Val}(S)\right\}$. The set $D(i)$ is often called the set of divisors of $s_{i}$, and its cardinality is denoted by $\nu_{i}=|D(i)|$.

Let $I$ be an ideal of $S$. We define the Frobenius number of the ideal $I$ to be the largest valuation of an element not belongs to $I$ denoted by $g(I)$, that is,

$$
g(I)=\max \{\operatorname{Val}(a) \mid a \notin I \text { and } a \in K\} .
$$

With the above notations, we have the following results.

Lemma 4.3. Let I be an $\mathfrak{n}$-primary ideal of $S$. Then we have

$$
\begin{aligned}
g(I) & =c(I)-1 \\
& =\max \{\operatorname{Val}(a) \mid a \notin I \text { and } a \in I: \mathfrak{n}\} .
\end{aligned}
$$

Proof. First, we show that $g(I)=c(I)-1$. Indeed, for all $a \in K$ such that $\operatorname{Val}(a)>c(I)-1$ then $\operatorname{Val}(a) \geq s_{n_{I}}$. It follows that $a \in S_{n_{I}}=I: \bar{S}$, whence $a \in I$. On the other hand, let $b \in K$ such that $\operatorname{Val}(b)=c(I)-1$. Then $\operatorname{Val}(b)<c(I)=s_{n_{I}}$. It implies that $b \notin S_{n_{I}}$. Hence, $b \notin I$. Therefore, we have $c(I)-1=g(I)$, as required.

Now let $g^{\prime}=\max \{\operatorname{Val}(a) \mid a \notin I$ and $a \in I: \mathfrak{n}\}$. Then it is clear that $g^{\prime}(I) \leq g(I)$. On the other hand, assume that there is an element $a \in K \backslash I$ such that $\operatorname{Val}(a)=g(I)$ but $a \notin I: \mathfrak{n}$. Then there exists $\alpha \in \mathfrak{n}$ such that $a \alpha \notin I$. It implies that $\operatorname{Val}(a \alpha) \leq \operatorname{Val}(a)$. Hence, $\operatorname{Val}(a)+\operatorname{Val}(\alpha) \leq \operatorname{Val}(a)$. We get $\operatorname{Val}(\alpha) \leq 0$ which is a contradiction to $\alpha \in \mathfrak{n}$. Thus, $g(I) \leq g^{\prime}(I)$, as required. 
Lemma 4.4. Let I be an $\mathfrak{n}$-primary ideal of $S$. Then the following assertions are true.

1) $\left.\left((I: \mathfrak{n}) \cap \mathfrak{n}^{s(S / I)}\right) \backslash I\right)=\{a \notin I \mid \operatorname{Val}(a)=g(I)\}$.

2) If $I=I_{1} \cap I_{2}$ then $g(I)=\max \left\{g\left(I_{1}\right), g\left(I_{2}\right)\right\}$. Moreover, if $I=\bigcap_{i=1}^{s} I_{i}$, where $I_{i}$ are irreducible then $g(I)=\max _{i=1}^{s}\left\{g\left(I_{i}\right)\right\}$.

3) We have

$$
\nu_{n_{I}} \leq \ell_{S}(S / I)+1
$$

Proof. 1) Recall that the degree of non-zero element $a \in S$ is defined by $\operatorname{deg} a=\max \{n \geq$ $\left.0 \mid a \in \mathfrak{n}^{n}\right\}$. Now, we show the following claim.

Claim 4.5. For all $a, b \in(I: \mathfrak{n}) \backslash I$, if $\operatorname{deg} b \leq \operatorname{deg} a$ then $a \bar{S} \subseteq b \bar{S}$.

Proof. Let $a, b \in(I: \mathfrak{n}) \backslash I$ such that $\operatorname{deg} b \leq \operatorname{deg} a$. Suppose $\operatorname{deg} b<\operatorname{deg} a$ but $a \bar{S} \nsubseteq b \bar{S}$. Then since $\bar{S}$ is a DVR, we have $b \bar{S} \subseteq a \bar{S}$. It follows that $b=a s$ for some $s \in \bar{S}$. Since $\operatorname{deg} b<\operatorname{deg} a$, we get $b \notin \mathfrak{n}^{\operatorname{deg}(a)}$ which implies that $a s \notin \mathfrak{n}^{\operatorname{deg}(a)}$. Hence, since $a \in \mathfrak{n}^{\operatorname{deg}(a)}$, one has

$$
\operatorname{Val}(a) \geq \operatorname{deg}(a)>\operatorname{Val}(a s)=\operatorname{Val}(a)+\operatorname{Val}(s) .
$$

Therefore, $\operatorname{Val}(s)<0$. This is impossible because $s \in \bar{S}$. Thus, if $\operatorname{deg} b<\operatorname{deg} a$ then $a \bar{S} \subseteq b \bar{S}$. Now if $\operatorname{deg}(a)=\operatorname{deg}(b)$ then $a \bar{S}=b \bar{S}$. Indeed, without loss of generality we may assume $b \bar{S} \subseteq a \bar{S}$. Then $b=a u$ for some $u \in \bar{S}$. Since $b \in \mathfrak{n}^{\operatorname{deg}(a)} \backslash \mathfrak{n}^{\operatorname{deg}(a)+1}$, we have

$$
\operatorname{deg}(a)+1>\operatorname{Val}(a)+\operatorname{Val}(u) \geq \operatorname{deg}(a) .
$$

Therefore, $\operatorname{Val}(a)+\operatorname{Val}(u)=\operatorname{deg}(a)$. We get $\operatorname{Val}(u)=0$. This implies that $u$ is invertible and hence $a \bar{S}=b \bar{S}$.

We now will show that

$$
(I: \mathfrak{n}) \cap \mathfrak{n}^{s(S / I)} \backslash I \subseteq\{a \in K \backslash I \mid \operatorname{Val}(a)=g(I)\} .
$$

Indeed, for all $a \in(I: \mathfrak{n}) \cap \mathfrak{n}^{s(S / I)} \backslash I$ and for all $b \in(I: \mathfrak{n}) \backslash I$, Proposition 2.1 implies that $\operatorname{deg}(b) \leq \operatorname{deg}(a)$. Hence, $a \bar{S} \subseteq b \bar{S}$ so that $\operatorname{Val}(b) \leq \operatorname{Val}(a)$. Therefore, by Lemma 4.3 we get $\operatorname{Val}(a)=g(I)$ as desired. For the reverse inclusion, we take $a \in K \backslash I$ such that $\operatorname{Val}(a)=g(I)$. Then we have $a \mathfrak{n} \subseteq I$. Because if otherwise then there exists $b \in \mathfrak{n}$ such that $a b \notin I$. It implies that

$$
\operatorname{Val}(a)+\operatorname{Val}(b)=\operatorname{Val}(a b) \leq \operatorname{Val}(a)
$$

whence $\operatorname{Val}(b)=0$. This is impossible because $b \in \mathfrak{n}$. Thus, $a \in I: \mathfrak{n}$. On the other hand, we take an element $b \in\left((I: \mathfrak{n}) \cap \mathfrak{n}^{s(S / I)}\right) \backslash I$, then $\operatorname{Val}(b)=g(I)$. Therefore $\operatorname{Val}(a)=\operatorname{Val}(b)$ and we get $a=b u$ for some a unit $u$ in $S$. Hence, $a \in\left((I: \mathfrak{n}) \cap \mathfrak{n}^{s(S / I)}\right) \backslash I$ as desired, that is, $(I: \mathfrak{n}) \cap \mathfrak{n}^{s(S / I)} \backslash I \supseteq\{a \in K \backslash I \mid \operatorname{Val}(a)=g(I)\}$.

2) Because $K \backslash I \supseteq\left(K \backslash I_{1}\right) \cup\left(K \backslash I_{2}\right)$, we get $g(I) \geq g\left(I_{i}\right)$ for all $i=1,2$. We suppose that $g(I)>g\left(I_{i}\right)$ for all $i=1,2$. Then there exists $a_{i} \in I_{i}$ such that $\operatorname{Val}\left(a_{i}\right)=g(I)$ for all $i=1,2$. Hence since $a_{2} \notin I_{1}$ we get $g(I)=\operatorname{Val}\left(a_{2}\right) \leq g\left(I_{1}\right)$ which is a contradiction. Thus $g(I)=\max \left\{g\left(I_{1}\right), g\left(I_{2}\right)\right\}$. Now we decompose each $I_{1}, I_{2}$ into the intersection of two ideals and appy the result above for $I_{1}, I_{2}$. Continuing the process, we get the latter assertion in 2 ). Here note that the process will stop when $I_{i}$ 's are irreducible. 
3) One has

$$
\begin{aligned}
\nu_{n_{I}} & =\mid\left\{s_{j} \mid s_{j} \leq s_{n_{I}} \text { and } s_{n_{I}-1}-s_{j} \in \operatorname{Val}(R)\right\} \mid \\
& \leq \mid\left\{s_{j} \mid s_{j} \leq s_{n_{I}} \text { and } s_{j}-e \notin I\right\} \mid+1 \\
& =\mid\left\{s_{j} \mid s_{j} \leq s_{n_{I}} \text { and } s_{j}-e \notin I\right\} \cup\left\{s_{n_{I}}-1\right\} \mid \\
& \leq \ell_{S}(S / I)+1 .
\end{aligned}
$$

Combining Proposition 2.1 and Lemma 4.4 we get the following result.

Corollary 4.6. $\{a \in K \backslash I \mid \operatorname{Val}(a)=g(I)\}=\{a \in K \backslash I \mid \operatorname{deg}(a)=s(S / I)\}$.

Theorem 4.7. Let I be an $\mathfrak{n}$-primary ideal of $S$. Then we have

$$
s(S / I) \leq s(S / I) e(\mathfrak{n}) \leq g(I) .
$$

Equality holds in 4.7.1 if and only if $S$ is a DVR.

Proof. Let $\operatorname{Ass}(S / I)=\left\{Q_{i}\right\}_{1 \leq i \leq n}$ be the set of associated prime ideals of $S / I$. Since $\mathfrak{n}^{s(S / I)} \nsubseteq$ $I,\left(\mathfrak{n}^{s(S / I)} \cap I+\mathfrak{n}^{s(S / I)+1}\right) / \mathfrak{n}^{s(S / I)+1}$ and $\left(\mathfrak{n}^{s(S / I)} \cap Q_{i}+\mathfrak{n}^{s(S / I)+1}\right) / \mathfrak{n}^{s(S / I)+1}$ are proper vector subspaces of $\mathfrak{n}^{s(S / I)} / \mathfrak{n}^{s(S / I)+1}$. Moreover, since $|S / \mathfrak{n}|=\infty$, we get

$$
\frac{\mathfrak{n}^{s(S / I)} \cap I+\mathfrak{n}^{s(S / I)+1}}{\mathfrak{n}^{s(S / I)+1}} \cup\left(\bigcup_{i=1}^{n} \frac{\mathfrak{n}^{s(S / I)} \cap Q_{i}+\mathfrak{n}^{s(S / I)+1}}{\mathfrak{n}^{s(S / I)+1}}\right) \neq \frac{\mathfrak{n}^{s(S / I)}}{\mathfrak{n}^{s(S / I)+1}} .
$$

Hence, we can choose $a \in \mathfrak{n}^{s(S / I)} \backslash\left(I \cup \bigcup_{i=1}^{n} Q_{i}\right)$ which is a superficial element of $\mathfrak{n}^{s(S / I)}$. Then since the analytic spread of $\mathfrak{n}^{s(S / I)}$ is $1, a R$ is a reduction of $\mathfrak{n}^{s(S / I)}$. Therefore, $e(a S)=$ $e\left(\mathfrak{n}^{s(S / I)}\right) \geq s(S / I) e(\mathfrak{n})$. On the other hand, we have

$$
e(a S)=\ell_{S}(S / a S)=\ell_{S}(S / a S)+\ell_{S}(\bar{S} / S)-\ell_{S}(a \bar{S} / a S)=\ell_{S}(\bar{S} / a \bar{S})=\operatorname{Val}(a) \leq g(I) .
$$

We therefore get the second inequality in the assertion.

The following lemma give an explicit formula for the number of the set of divisors of $s_{i}$.

Lemma 4.8. [BA13, Lemma 51] For each $i \in \mathbb{N}$, let $\delta(i)$ be the number of gaps in the interval from 1 to $s_{i}-1$ and let $G(i)$ be the number of pairs of gaps whose sum equals $s_{i}$. Then, $\nu_{i}=i-\delta(i)+G(i)+1$.

We have the following theorem which is an extension of a result of M. Bras-Amorós [BA19, Theorem 1].

Theorem 4.9. Let $I$ be an $\mathfrak{n}$-primary ideal of $S$. Then

$$
g(I)+1 \leq \ell_{S}(S / I)+2 \delta .
$$

Proof. It is straightforward to see that the intersection of two ideals satisfying the result also satisfies the result. Now, by Lemma 4.42 ), it will be enough to show that the result holds for the irreducible ideal $I$. By Lemma 4.43 ), it will be enough to show that $\nu_{n_{I}}-1+2 \delta \geq c(I)$. Indeed, since $s_{n_{I}} \geq c$, one has $\delta\left(n_{I}\right)=\delta, s_{n_{I}}=n_{I}+\delta$, and as a consequence of Lemma 4.8,

$$
\nu_{n_{I}}-1+2 \delta=\left(n_{I}-\delta+G\left(n_{I}\right)+1\right)-1+2 \delta=n_{I}+\delta+G\left(n_{I}\right)=s_{n_{I}}+G\left(n_{I}\right) \geq s_{n_{I}} .
$$


Corollary 4.10. Let $I$ be an $\mathfrak{n}$-primary ideal of $S$. Then

$$
\ell_{S}\left(I / \mathfrak{C}_{I}\right) \leq \delta
$$

Proof. Thanks to Theorem 4.9 we have

$$
\ell_{S}\left(\bar{S} / \mathfrak{C}_{I}\right)=\delta+\ell_{S}\left(R / \mathfrak{C}_{I}\right)=c(I) \leq \ell_{S}(S / I)+2 \delta .
$$

Therefore, $\ell_{S}\left(R / \mathfrak{C}_{I}\right) \leq \ell_{S}(S / I)+\delta$. Hence, $\ell_{S}\left(I / \mathfrak{C}_{I}\right) \leq \delta$.

Definition 4.11. Let $(S, \mathfrak{n})$ denote a Cohen-Macaulay local $\operatorname{ring}$ with $\operatorname{dim} S=1$. Let $K$ be the total quotient ring of $S$ and $\bar{S}$ the integral closure of $S$ in $K$. An $\mathfrak{n}$-primary ideal $I$ of $S$ is called maximum sparse, if $\ell_{S}\left(I / I:_{K} \bar{S}\right)=\ell_{S}(\bar{S} / S)$.

Example 4.12. Let $S=k[[H]]=k\left[\left[t^{s} \mid s \in H\right]\right] \subseteq k[[t]]$ be a semigroup ring of a numerical semigroup $H$ and $I$ be an ideal of $S$. Then $\operatorname{Val}(S)=H$ and $\operatorname{Val}(I)$ is an ideal of $H$. It is easy to see that if $I$ is maximum sparse then $\operatorname{Val}(I)$ is also a maximum sparse ideal. The converse also holds.

Example 4.13. Let $S=k\left[\left[t^{4}, t^{6}+t^{7}, t^{15}\right]\right] \subseteq \bar{S}=k[[t]]$, where char $(k) \neq 2$. It is not difficult to see that $\operatorname{Val}(S)=\langle 4,6,13,15\rangle$. Let $I=\left(t^{12}-t^{16}, t^{14}+t^{15}+t^{16}+t^{17}\right) S$. By a direct computation, we get $\ell_{S}(\bar{S} / S)=7, g(I)=23$ and $\ell_{S}(S / I)=10$. Therefore,

$$
g(I)+1=2 \ell_{S}(\bar{S} / S)+\ell_{S}(S / I) .
$$

Hence, $I$ is a maximal sparse ideal. Notice that $S$ is not a numerical semigroup ring.

Remark 4.14. Let $S$ be as in Setting 4.1. Then $S$ always contains a maximal sparse ideal. Indeed, if we let $\operatorname{Val}(S)=\left\{\lambda_{0}<\lambda_{1}<\cdots\right\}$ then since $\sharp(\mathbb{N} \backslash \operatorname{Val}(S))<\infty$, there exists $i>0$ such that $\lambda_{i}$ less than twice of the Frobenius number of $\operatorname{Val}(S)$. Then $G(i)=0$ so that $I:=\operatorname{Val}(S) \backslash D(i)$ is a maximum sparse ideal of $\operatorname{Val}(S)$. Hence, the preimage $\mathrm{Val}^{-1}(I)$ is a maximum sparse ideal in $S$.

Notice that the canonical module of $S$ in Example 4.13 is $\omega_{S}=R+\frac{t^{2}}{1-t} R$ and $I=$ $\left(t^{12}-t^{16}\right) \omega_{S}$ so that $I \cong \omega_{S}$. Hence, $I$ is also a canonical ideal of $S$. In general, this is also true for every maximum ideal.

Lemma 4.15. If $I$ is a maximum sparse ideal of $S$ then $I$ is a canonical ideal.

Proof. Suppose that $I$ is a maximum sparse ideal of $S$. Since $I$ is an $\mathfrak{n}$-primary ideal and $S$ is a one-dimensional integral domain, we get $0<\operatorname{depth}_{S}(I) \leq \operatorname{dim}_{S} I \leq \operatorname{dim} S=1$. Hence, $I$ is a Cohen-Macaulay $S$-module of dimension one. On the other hand, since $g(I)+1=$ $\ell_{S}(S / I)+2 \ell_{S}(\bar{S} / S)$. It implies that

$$
g(\operatorname{Val}(I))+1=|\operatorname{Val}(S) \backslash \operatorname{Val}(I)|+2 \ell_{S}(\bar{S} / S)
$$

Thus, $\operatorname{Val}(I)$ is a maximum sparse ideal of the semigroup $\operatorname{ring} \operatorname{Val}(S)$. Therefore, $\operatorname{Val}(I)=$ $\operatorname{Val}(S) \backslash D(i)$ for some $i$ such that $G(i)=0$. We get that $\operatorname{Val}(I)$ is irreducible (by [BK10, Proposition 1]). Hence, $I$ is irreducible as well (by [BK10, Theorem 3]). We get $\ell_{S}(I$ : $\mathfrak{n} / I)=1$. Therefore, thanks to [HK71, Satz 3.3], $I$ is a canonical ideal of $S$.

Now, let us give a characterization for the Gorensteinness of $S$. 
Theorem 4.16. The following statements are equivalent.

1) $S$ is Gorenstein.

2) There is a maximum sparse ideal I of $S$ such that $I$ is a principal ideal.

3) Every canonical ideal of $S$ is maximum sparse.

Proof. 1) $\Rightarrow 2$ ) Suppose that $S$ is Gorenstein. By Remark 4.14 we take $I$ to be a maximum sparse ideal. Then, Lemma 4.15 says that $I$ is a canonical ideal of $S$. Since the residue field $S / \mathfrak{n}$ is infinite, there exists $Q=(a)$ a reduction of $I$. Then since $S$ is Gorenstein, we have $I=Q$ (by [GMP13, Lemma 3.7]). Hence, $I$ is principal.

$2) \Rightarrow 1$ ): Suppose $I=a S$ is a maximum sparse ideal of $S$. Then $I$ is irreducible. Hence, since $a$ is regular on $S$, we get

$$
r_{S}(S)=r_{S}(S / a S)=r_{S}(S / I)=\ell_{S}(I: \mathfrak{n} / I)=1 .
$$

Therefore, $S$ is Gorenstein.

$1) \Rightarrow 3$ ) Suppose that $S$ is Gorenstein and $I$ is a canonical ideal of $S$. Since $I \cong S$, we have $I=a S$ for some $a \in I$. Note that $a$ is unit in $Q(S)$, one has

$$
\ell_{S}(I /(I: \bar{S}))=\ell_{S}(a S /(a S: \bar{S}))=\ell_{S}(a S / a(S: \bar{S}))=\ell_{S}(S / \mathfrak{C}) .
$$

Hence, on the one hand we have $\ell_{S}(S / \mathfrak{C}) \leq \ell_{S}(\bar{S} / S)$ (by Theorem 4.9). On the other hand, $\ell_{S}(\bar{S} / S) \leq \ell_{S}(S / \mathfrak{C})$ (by [BH92, Theorem 1]). It implies that $\ell_{S}(\bar{S} / S)=\ell_{S}(S / \mathfrak{C})$. Thus, $\ell_{S}(I /(I: \bar{S}))=\ell_{S}(\bar{S} / S)$ so that $I$ is maximum sparse.

$3) \Rightarrow 1$ ) Taking the canonical module $\omega_{S}$ as in Lemma 2.4. If $\omega_{S}=S$ then it is clear that $S$ is Gorenstein. If otherwise, we may let $\omega_{S}=S+\sum_{i=1}^{n} \frac{a_{i}}{b_{i}} S$ for some a positive integer $n$ and some $a_{i}, b_{i} \in S, b_{i} \neq 0$ for all $1 \leq i \leq n$. Let $x=b_{1} b_{2} \ldots b_{n}$. Then $x \omega_{S}$ is a canonical ideal of $S$. Let $x z S$ is a minimal reduction of $x \omega_{S}$, where $z \in \omega_{S}$. Then by ([Del94, Lemma 1.11]) we have $\ell_{S}\left(z \bar{S} / \omega_{S}\right)=\ell_{S}(S / \mathfrak{C})$. Moreover, since $\mathfrak{C}$ is an ideal of both $S$ and $\bar{S}$, we have

$$
x \omega_{S} \mathfrak{C}=x \omega_{S} \bar{S} \mathfrak{C}=x z \bar{S} \mathfrak{C}=x z \mathfrak{C} .
$$

Therefore,

$$
\begin{aligned}
\ell_{S}(\bar{S} / S) & =\ell_{S}(x z \bar{S} / x z S) \\
& =\ell_{S}\left(x z \bar{S} / x \omega_{S}\right)+\ell_{S}\left(x \omega_{S} / x z S\right) \\
& =\ell_{S}(S / \mathfrak{C})+\ell_{S}\left(x \omega_{S} / x z S\right) \\
& =\ell_{S}\left(x \omega_{S} / x z \mathfrak{C}\right) \\
& =\ell_{S}\left(x \omega_{S} / x \omega_{S} \mathfrak{C}\right) \\
& \leq \ell_{S}\left(x \omega_{S} / \mathfrak{C}_{x \omega_{S}}\right)\left(\text { because } x \omega_{S} \mathfrak{C} \subseteq \mathfrak{C}_{x \omega_{S}}\right)
\end{aligned}
$$

On the other hand, by the assumption, $x \omega_{S}$ is maximum sparse. Therefore we obtain the equality above. Hence, $x \omega_{S} \mathfrak{C}=\mathfrak{C}_{x \omega_{S}}$. It follows that

$$
x z \mathfrak{C}=x \omega_{S}: \bar{S}=x\left(\omega_{S}: \bar{S}\right)=x \mathfrak{C}
$$

We get $z \mathfrak{C}=\mathfrak{C}$. Note that $z$ is in $S$. If $z \in \mathfrak{m}$ then by Nakayama's lemma we get $\mathfrak{C}=0$ which is a contradiction. Therefore, $z$ is unit, whence $S=x \omega$. Consequently, $S$ is Gorenstein. 
We consider Theorem 4.16 in the context of numerical semigroup rings. Let $H$ be a numerical semigroup. A relative ideal $I$ of $H$ is called a canonical ideal of $H$ if $I-(I-J)=J$ for every relative ideal $J$. A typical example of canonical ideals is the ideal $\Omega=\{g(S)-a \mid$ $a \in \mathbb{Z} \backslash S\}$ which is called the standard canonical ideal of $S$. It is easy to see that if $S=k[[H]]$ be the numerical semigroup ring of $H$, then an ideal $I$ of $S$ is maximum sparse if and only if $\operatorname{Val}(I)$ is maximum sparse of $H$. The ideal $I$ is a canonical ideal of $S$ if and only if $\operatorname{Val}(I)$ is a canonical ideal of $H$ (cf. [Jäg77]). Moreover, $S$ is Gorenstein if and only if $H$ is symmetric (cf. [Kun70]). Therefore, by applying Theorem 4.16 for the case $S=k[[H]]$, then we immediately get the following result in numerical semigroups.

Corollary 4.17. Let $H$ be a numerical semigroup. Then the following assertions are equivalent.

1) $H$ is symmetric.

2) There is a maximum sparse ideal I of $H$ such that $I$ is a principal ideal.

3) Every canonical ideal of $H$ is maximum sparse.

Now we define sparse stretched rings which is a special class of canonical stretched rings.

Definition 4.18. We say that $(S, \mathfrak{n})$ is a sparse stretched ring, if there is a maximum sparse ideal $I \subseteq \mathfrak{n}^{2}$ of $S$ such that $S / I$ is a stretched ring.

Proposition 4.19. If $S$ is a sparse stretched ring then $S$ is a canonical stretched ring.

Proof. It is now immediate from Lemma 4.15 and the definition of sparse stretched rings.

Note that the reverse implication may not be true (see Example 5.2).

Theorem 4.20. Assume that the Hilbert function of $S$ is non-decreasing. Then the following statements are equivalent.

1) $S$ is a sparse stretched ring.

2) There exists an $\mathfrak{n}$-primary ideal $I \subseteq \mathfrak{n}^{2}$ such that $\mu\left(\mathfrak{n}^{v_{S}(I)} / I\right)=1, I: \mathfrak{n} \subseteq \mathfrak{n}^{v_{S}(I)}$ and

$$
s(I)(\operatorname{Val}(z)-1)=2 \delta+\operatorname{edim}(S)-1
$$

for some $z \in \mathfrak{n} \backslash \mathfrak{n}^{v_{S}(I)+1}$ and $z^{2} \notin I$.

When this is the case, $v_{S}(I)=2$.

Proof. 1) $\Rightarrow 2$ ) Since $S$ is a sparse stretched ring, there exists an irreducible ideal $I \subseteq \mathfrak{n}^{2}$ of $S$ such that $S / I$ is a stretched ring. By Corollary 3.4, we have $v_{S}(I)=2, \mu\left(\mathfrak{n}^{v_{S}(I)} / I\right)=1$ and $I: \mathfrak{n} \subseteq \mathfrak{n}^{v_{S}(I)}$.

Let $R=S / I$ and $\mathfrak{m}=\mathfrak{n} / I$. Then, by Lemma 2.4 , there exists a basis $z_{1}, \ldots, z_{\operatorname{edim}(S)}$ for $\mathfrak{n}$ such that $\mathfrak{m}^{2}=z_{1}^{2} R$. Thus, $\mathfrak{m}^{s(R)}=z_{1}^{s(R)} R$. By the definition, we have

$$
g(I)=s(R) \operatorname{Val}\left(z_{1}\right) .
$$

Since $S / I$ is a stretched ring and $I$ is a maximum sparse ideal, we have

$$
\begin{aligned}
s(R)\left(\operatorname{Val}\left(z_{1}\right)-1\right) & =g(I)-s(R) \\
& =2 \delta+\ell_{S}(S / I)-s(R)-1 \\
& =2 \delta+\operatorname{edim}(S)-1 .
\end{aligned}
$$


$2) \Rightarrow 1$ ) Let $R=S / I$ and $\mathfrak{m}=\mathfrak{n} / I$. It follows from $\mu\left(\mathfrak{m}^{v_{S}(I)}\right)=1$ and Corollary 3.4 that the Hilbert function of $R$ is given by 3.3.2. Therefore, by Theorem 3.4 in [Sha14], we have

$$
h_{S}\left(v_{S}(R)-1\right)-h_{S}(1)+1 \leq \operatorname{dim}_{k}\left(0:_{R} \mathfrak{m}\right) .
$$

Moreover, since $0:_{R} \mathfrak{m} \subseteq \mathfrak{m}^{v_{S}(R)}$ and $\mu\left(\mathfrak{m}^{v_{S}(I)}\right)=1$, we have $\operatorname{dim}_{k}\left(0:_{R} \mathfrak{m}\right)=1$. Then, $h_{S}\left(v_{S}(R)-1\right)-h_{S}(1) \leq 0$. Since the Hilbert function $h_{S}$ of $S$ is non-decreasing, we have $v_{S}(R)=2$. Then, by Corollary 3.4, $S / I$ is a stretched ring.

Since $z \notin \mathfrak{n}^{v_{S}(I)+1}, z^{2} \notin I$ and $\mathfrak{m}^{2}$ is principal ideal, $\mathfrak{m}^{s(R)}=z_{1}^{s(R)} R$. By the definition, we have

$$
\begin{aligned}
g(I) & =s(R) \operatorname{Val}(z) \\
& =2 \delta+\operatorname{edim}(S)-1+s(R) \\
& =2 \delta+\ell_{S}(S / I)-1 .
\end{aligned}
$$

Hence, $I$ is a maximum sparse ideal of $S$.

Example 4.21. We look at the local ring

$$
S=k\left[\left[t^{6}, t^{7}, \ldots, t^{11}\right]\right]
$$

in the formal power series ring $k[[t]]$ over a field $k$. We put $I=\left(t^{12}, t^{13}, t^{14}, t^{15}, t^{16}\right)$. Then $g(I)=17, \ell_{S}(S / I)=8$ and $\delta=5$. Thus, we have

$$
g(I)=\ell_{S}(S / I)+2 \delta-1,
$$

and $I$ is a maximum sparse ideal of $S$. Moreover $S / I$ is an Artinian stretched ring, because the Hilbert function of $S / I$ is

$$
h_{S / I}(i)= \begin{cases}1 & \text { if } i=0, \\ 6 & \text { if } i=1, \\ 1 & \text { if } i=2, \\ 0 & \text { if } i \geq 3 .\end{cases}
$$

Hence, $S$ is a sparse stretched ring.

Example 4.22. Let $S=k\left[\left[t^{4}, t^{6}+t^{7}, t^{15}\right]\right] \subseteq \bar{S}=k[[t]]$, where $\operatorname{char}(k) \neq 2$. We have the value semigroup $\operatorname{Val}(S)=\langle 4,6,13,15\rangle$. By a direct computation, we get $\ell_{S}(\bar{S} / S)=7$ and $\operatorname{edim}(S)=3$. Therefore $2 \ell_{S}(\bar{S} / S)+\operatorname{edim}(S)-1=16$. Assume that $S$ is a sparse stretched ring. Then, by Theorem 4.20, there exist $I \subseteq \mathfrak{n}^{2}$ and $z \in \mathfrak{n} \backslash \mathfrak{n}^{v_{S}(I)+1}$ such that $z^{2} \notin I$ and $s(I)(\operatorname{Val}(z)-1)=16$. Hence, since $2,3,5,9 \notin \operatorname{Val}(S)$, we must have $\operatorname{Val}(z)=17$ which implies $s(I)=1$. It follows that $I=\mathfrak{n}^{2}$ so that $z^{2} \in I$ which is a contradiction. Therefore, $S$ is not a sparse stretched ring.

\section{CANONicAl StRetched PROPERTy of 3-Generated Numerical SEMigroup Rings}

In this section, we analyze the semigroup ring $k[H]$ of a 3-generated numerical semigroup $H$ with $k$ is a field. Let $a_{1}, a_{2}, a_{3} \in \mathbb{Z}$ and assume that $0<a_{1}<a_{2}<a_{3}$ with $\operatorname{GCD}\left(a_{1}, a_{2}, a_{3}\right)=$ 1. Let $H$ be the numerical semigroup generated by $a_{1}, a_{2}, a_{3}$, that is, $H=\left\langle a_{1}, a_{2}, a_{3}\right\rangle:=$ $\left\{c_{1} a_{1}+c_{2} a_{2}+c_{3} a_{3} \mid 0 \leq c_{i} \in \mathbb{Z}, 1 \leq i \leq 3\right\}$. Let $k[t]$ denotes the polynomial ring and put $T=k\left[t^{a_{1}}, t^{a_{2}}, t^{a_{3}}\right] \subseteq k[[t]]$. Then, $T$ is a one-dimensional graded domain with $\bar{T}=k[t]$, where 
$\bar{T}$ stands for the normalization of $T$. Let $M=\left(t^{a_{1}}, t^{a_{2}}, t^{a_{3}}\right)$ denote the maximal ideal of $T$ generated by $t^{a_{i}}$ 's. In this section, we explore the local $\operatorname{ring} S=T_{M}$ and eventually answer the question of when $S=k\left[\left[t^{a_{1}}, t^{a_{2}}, t^{a_{3}}\right]\right]$ is a sparse/canonical stretched ring. Throughout, we assume that $S$ is not a Gorenstein ring. Let $U=k[[X, Y, Z]]$ be the polynomial ring and regard $U$ as a $\mathbb{Z}$-graded ring with $U_{0}=k, \operatorname{deg} X=a_{1}, \operatorname{deg} Y=a_{2}$, and $\operatorname{deg} Z=a_{3}$. Let $\varphi: U \rightarrow S$ be the $k$-algebra homomorphism defined by $\varphi(X)=t^{a_{1}}, \varphi(Y)=t^{a_{2}}$, and $\varphi(Z)=$ $t^{a_{3}}$. Hence, $\operatorname{Im} \varphi=S$. We put $J=\operatorname{Ker} \varphi$. Then, because $S$ is not a Gorenstein ring, thanks to [Her70], the ideal $J$ is generated by the maximal minors of the matrix (which is called the Herzog matrix)

$$
\left(\begin{array}{lll}
X^{\alpha} & Y^{\beta} & Z^{\gamma} \\
Y^{\beta^{\prime}} & Z^{\gamma^{\prime}} & X^{\alpha^{\prime}}
\end{array}\right),
$$

where $0<\alpha, \beta, \gamma, \alpha^{\prime}, \beta^{\prime}, \gamma^{\prime} \in \mathbb{Z}$. Let us call this matrix the Herzog matrix of $H$. Let $\Delta_{1}=Z^{\gamma+\gamma^{\prime}}-X^{\alpha^{\prime}} Y^{\beta}, \Delta_{2}=X^{\alpha+\alpha^{\prime}}-Y^{\beta^{\prime}} Z^{\gamma}$, and $\Delta_{3}=Y^{\beta+\beta^{\prime}}-X^{\alpha} Z^{\gamma^{\prime}}$. Then $J=\left(\Delta_{1}, \Delta_{2}, \Delta_{3}\right)$ and $S \cong U / J$.

We are in a position to summarize these arguments.

Theorem 5.1. Let $S=k\left[\left[t^{a_{1}}, t^{a_{2}}, t^{a_{3}}\right]\right]$ is a numerical semigroup ring with embedding dimension three. Then $S$ is always a canonical stretched ring.

Proof. Firstly, we see that the Herzog matrix of $S$ do not have the form

$$
\left(\begin{array}{lll}
X & Y & Z \\
Y & Z & X
\end{array}\right) .
$$

Indeed, suppose $S \cong U / \mathrm{I}_{2}\left(\begin{array}{lll}X & Y & Z \\ Z\end{array}\right)$. Then one has (see [Her70])

$$
\left\{\begin{array}{l}
2 a_{1}=a_{2}+a_{3} \\
2 a_{2}=a_{1}+a_{3} \\
2 a_{3}=a_{1}+a_{2}
\end{array} .\right.
$$

Hence, $a_{1}=a_{2}=a_{3}=1$ (by $\operatorname{gcd}\left(a_{1}, a_{2}, a_{3}\right)=1$ ). It implies that $S=k[[t]]$. Therefore, $k[[t]] \cong k[[X, Y, Z]] /\left(X^{2}-Y Z, Y^{2}-X Z, Z^{2}-X Y\right)$. It is clear that the right hand side is not a DVR (because it has embedding dimension 3) which is a contradiction to the left hand side.

Hence, $\max \left\{\alpha+\alpha^{\prime}, \beta+\beta^{\prime}, \gamma+\gamma^{\prime}\right\} \geq 3$. Then, we may assume that $\alpha+\alpha^{\prime} \geq 3$. Let $I=$ $\left(X Y, Y Z, Z X, X^{s}+Y^{2}, X^{s}+Z^{2}\right)$ for $2 \leq s \leq \alpha+\alpha^{\prime}-1$. Then $X^{s+1}=X\left(X^{s}+Y^{2}\right)-X Y^{2} \in I$, $Y^{3}=Y\left(X^{s}+Y^{2}\right)-X^{s} Y \in I$ and $Z^{3}=Z\left(X^{s}+Z^{2}\right)-X^{s} Z \in I$. Thus $J \subseteq I$.

Let $\mathfrak{n}=(X, Y, Z) U$ and $\mathfrak{m}=\mathfrak{n} / J$. Then $I \subseteq \mathfrak{n}^{2}, I: \mathfrak{n}=\left(X Y, Y Z, Z X, X^{s}, Y^{2}, Z^{2}\right)$ and so $\ell_{U}((I: \mathfrak{n}) / I)=1$. Hence, for all $2 \leq s \leq \alpha+\alpha^{\prime}-1, I$ is a canonical ideal of $U$. Moreover, the Hilbert function of $U / I$ is

\begin{tabular}{|c|c|c|c|c|c|}
\hline 0 & 1 & 2 & $\ldots$ & $s$ & $s+1$ \\
\hline 1 & 3 & 1 & $\ldots$ & 1 & 0 \\
\hline
\end{tabular}

Hence, $S$ is a canonical stretched ring. 
We close this paper with the following two examples of canonical stretched rings. One of them is not a sparse stretched ring and the other is not a stretched ring.

Example 5.2. Let $S=k\left[\left[t^{4}, t^{5}, t^{7}\right]\right]$ is a numerical semigroup ring over a field $k$ with the maximal ideal $\mathfrak{n}=\left(t^{4}, t^{5}, t^{7}\right)$. Thanks to Theorem 5.1, $S$ is a canonical stretched ring. But $S$ is not a sparses stretched ring. Indeed, assume that $S$ is a sparse stretched ring. Then, by Theorem 4.20, there exist $I \subseteq \mathfrak{n}^{2}$ and $z \in \mathfrak{n} \backslash \mathfrak{n}^{v_{S}(I)+1}$ such that $z^{2} \notin I$ and $s(I)(\operatorname{Val}(z)-1)=$ $2 \delta+\operatorname{edim}(S)-1$. Since $\delta=4, \operatorname{edim} S=3, s(I)(\operatorname{Val}(z)-1)=2 \delta+\operatorname{edim}(S)-1=10$. Thus since $2,3,6 \notin \operatorname{Val}(S)$, we must have $\operatorname{Val}(z)=11$ and $s(I)=1$. It follows that $I=\mathfrak{n}^{2}$ and so $z^{2} \in I$, which is a contradiction. Hence $S$ is not a sparses stretched ring.

Example 5.3. Let $S=k\left[\left[t^{5}, t^{6}, t^{13}\right]\right]$ is a numerical semigroup ring over a field $k$ with the maximal ideal $\mathfrak{n}=\left(t^{5}, t^{6}, t^{13}\right)$. Then by Theorem 5.1, $S$ is a canonical stretched ring. But $S$ is not a stretched ring. In fact, we can check that $\mathfrak{n}$ contains a unique minimal reduction $J=\left(t^{5}\right)$. Notice that $\ell_{S}\left(\frac{\mathfrak{n}^{2}+J}{\mathfrak{n}^{3}+J}\right)=2$.

\section{ACKNOWLEDGMENTS}

The second author was partially supported by the Vietnam National Foundation for Science and Technology Development (NAFOSTED) under grant number 101.04-2019.309. The third author was partially supported by the Alexander von Humboldt Foundation and the Vietnam National Foundation for Science and Technology Development (NAFOSTED) under grant number 101.04-2019.309. The authors would like to thank the referee for the valuable comments to improve this article. The Theorem 5.1 is largely due to the inspiring suggestions of Naoyuki Matsuoka.

\section{REFERENCES}

[BA13] M Bras-Amorós, Numerical semigroups and codes. In Algebraic Geometry Modeling in Information Theory, Volume 8 of Ser. Coding Theory Cryptol.; World Science Publisher: Hackensack, NJ, USA, (2013), 167-218. $\uparrow 12$

[BA19] M. Bras-Amorós, Ideals of numerical semigroups and error-correcting codes, Symmetry 11 (2019), 1406. $\uparrow 9,12$

[BALVO14] M. Bras-Amorós, K. Lee, and A. Vico-Oton, New Lower Bounds on the Generalized Hamming Weights of $A G$ Codes, IEEE Trans. on Information Theory, 60 (2014), no. 10, 5930-5937. $\uparrow 9$

[BH92] W. C. Brown and J. Herzog, One dimensional local rings of maximal and almost maximal length, J. Algebra 151 (1992), 332-347. $\uparrow 4,14$

[BK10] V. Barucci and F. Khouja, Irreducibility of ideals in a one-dimensional analytically irreducible ring, C.I.R.M. (2010). $\uparrow 13$

[Del94] D. Delfino, On the inequality $\lambda(\bar{R} / R) \leq t(R) \lambda(R / \mathfrak{C})$ for one-dimensional local rings, J. Algebra 1994 (1994), 332-342. $\uparrow 14$

[EV08] J. Elias and G. Valla, Structure theorems for certain Gorenstein ideals, Michigan Math. J. 57 (2008), 269-292. $\uparrow 1$

[GMP13] S. Goto, N. Matsuoka, and T. T. Phuong, Almost Gorenstein rings, J. Algebra 379 (2013), 355-381. 4 , 14

[Her70] J. Herzog, Generators and relations of Abelian semigroups and semigroup rings, Manuscripta Math. 3 (1970), 175-193. $\uparrow 17$

[HK71] J. Herzog and E. Kunz, Der kanonische Modul eines Cohen-Macaulay-Rings, Vol. 238, Lecture Notes in Math., Sppringer-Verlag, 1971. $\uparrow 1,3,4,13$ 
[Jäg77] Jäger, Läangenberechnung und kanonische ideale in eindimensionalen ringen, Arch. Math. 29 (1977). $\uparrow 15$

[Kun70] E. Kunz, The value semigroup of a one dimensional Gorenstein ring, Proc. Amer. Math. Soc. 25 (1970), 748-751. $\uparrow 15$

[Mat71] T. Matsuoka, On the degree of singularity of one-dimensional analytically irreducible Noetherian local rings, J. Math. Kyoto Univ. (1971), 485-494. $\uparrow 9$

[Ooi82] A. Ooishi, Castelnuovo's regularity of graded rings and modules, Hiroshima Math. J. 12 (1982), 627-644. $\uparrow 4$

[Sal79] J. D. Sally, Stretched Gorenstein rings, J. London Math. Soc. 20 (1979), no. 2, 19-26. $11,3,7$

[Sha14] L. Sharifan, A class of Artinian local rings of homogeneous type, Bull. Iranian Math. Soc. 40 (2014), no. 1, 157-181. $\uparrow 8,16$

The Department of Mathematics, Thai Nguyen University of education. 20 Luong Ngoc Quyen Street, Thai Nguyen City, Thai Nguyen Province, Viet Nam.

Email address: hangnthianh@gmail.com

Department of Mathematics, Hanoi Pedagogical University 2, Vinh Phuc, Viet Nam

Email address: dovankien@hpu2.edu.vn

Mathematik und Informatik, Universität des SaArlandes, Campus E2 4, D-66123 SaARbrüCKen, Germany

Institute of Mathematics, VAST, 18 Hoang Quoc Viet Road, 10307 Hanoi, Viet Nam

Thang Long Institute of Mathematics and Applied Sciences, Hanoi, Vietnam

Email address: hoang@math.uni-sb.de, hltruong@math.ac.vn, truonghoangle@gmail.com 